\title{
Long-Term Homeostasis of Extracellular Glutamate in the Rat Cerebral Cortex across Sleep and Waking States
}

\author{
Michael B. Dash, ${ }^{1,2}$ Christopher L. Douglas, ${ }^{1}$ Vladyslav V. Vyazovskiy, ${ }^{1}$ Chiara Cirelli, ${ }^{1}$ and Giulio Tononi ${ }^{1}$ \\ ${ }^{1}$ Department of Psychiatry, and ${ }^{2}$ Neuroscience Training Program, University of Wisconsin-Madison, Madison, Wisconsin 53719
}

\begin{abstract}
Neuronal firing patterns, neuromodulators, and cerebral metabolism change across sleep-waking states, and the synaptic release of glutamate is critically involved in these processes. Extrasynaptic glutamate can also affect neural function and may be neurotoxic, but whether and how extracellular glutamate is regulated across sleep-waking states is unclear. To assess the effect of behavioral state on extracellular glutamate at high temporal resolution, we recorded glutamate concentration in prefrontal and motor cortex using fixedpotential amperometry in freely behaving rats. Simultaneously, we recorded local field potentials (LFPs) and electroencephalograms (EEGs) from contralateral cortex. We observed dynamic, progressive changes in the concentration of glutamate that switched direction as a function of behavioral state. Specifically, the concentration of glutamate increased progressively during waking $(0.329 \pm 0.06 \% / \mathrm{min})$ and rapid eye movement $(\mathrm{REM})$ sleep $(0.349 \pm 0.13 \% / \mathrm{min})$. This increase was opposed by a progressive decrease during non-REM (NREM) sleep $(0.338 \pm 0.06 \% / \mathrm{min})$. During a $3 \mathrm{~h}$ sleep deprivation period, glutamate concentrations initially exhibited the progressive rise observed during spontaneous waking. As sleep pressure increased, glutamate concentrations ceased to increase and began decreasing despite continuous waking. During NREM sleep, the rate of decrease in glutamate was positively correlated with sleep intensity, as indexed by LFP slow-wave activity. The rate of decrease doubled during recovery sleep after sleep deprivation. Thus, the progressive increase in cortical extrasynaptic glutamate during EEG-activated states is counteracted by a decrease during NREM sleep that is modulated by sleep pressure. These results provide evidence for a long-term homeostasis of extracellular glutamate across sleep-waking states.
\end{abstract}

Key words: glutamate; in vivo amperometry; sleep; rat; cerebral cortex; EEG; slow-wave activity

\section{Introduction}

The transition between wakefulness, non-REM (NREM), and REM sleep is accompanied by neuromodulatory changes. Acetylcholine, norepinephrine, serotonin, histamine, and hypocretin levels are high in wakefulness and low in NREM sleep, and noradrenergic, serotonergic and histaminergic cells become silent during REM sleep (Jones, 2005). After neuromodulation, the electroencephalograms (EEG) and the underlying patterns of neuronal activity also change, especially in cerebral cortex. The low-voltage fast-activity EEG of wakefulness and REM sleep is called "activated" because neurons are steadily depolarized and fire tonically (Steriade et al., 2001). During NREM sleep, when large slow waves appear in the EEG, neurons exhibit periods of hyperpolarization lasting hundreds of milliseconds, during which synaptic activity ceases altogether (Steriade et al., 2001). Accompanying these changes in neuronal activity are variations in energy metabolism. In waking and REM sleep overall brain metabolism is high in both animals and humans (Madsen et al.,

Received Nov. 13, 2008; accepted Dec. 9, 2008.

This work was supported by the National Institute of Mental Health (Grant P20 MH077967 to C.C.) and by the National Institutes of Health Director's Pioneer Award (to G.T.). We thank Francois Pomerleau at the University of Kentucky for technical advice.

Correspondence should be addressed to Dr. Giulio Tononi, Department of Psychiatry, University of WisconsinMadison, 6001 Research Park Boulevard, Madison, WI 53719. E-mail: gtononi@wisc.edu.

DOI:10.1523/JNEUROSCI.5486-08.2009

Copyright $\odot 2009$ Society for Neuroscience $\quad$ 0270-6474/09/290620-10\$15.00/0
1991; Maquet, 1995). During deep NREM sleep, instead, cerebral glucose metabolism is $\sim 30-40 \%$ lower than during waking (Kennedy et al., 1982; Maquet, 1995), and so are cerebral blood flow and oxygen metabolism ( -18 and $-25 \%$, respectively) (Madsen et al., 1991).

Neuromodulators and changes in firing pattern affect glutamate release and clearance (Tzingounis and Wadiche, 2007). Moreover, most of the brain energy budget is required to sustain synaptic activity at glutamatergic synapses (Attwell and Laughlin, 2001). Thus, glutamatergic activity may also change as a function of behavioral state, although it is unclear how. Knowing whether and how it changes would be important, because glutamatergic signaling is critical for brain function at many levels, including information transmission, plasticity, and neurotoxicity. Unfortunately, the available evidence is limited and inconsistent. Some found increased glutamate release during EEG-activated states in cortex (Jasper et al., 1965; Lopez-Rodriguez et al., 2007) and anterior hypothalamus (Azuma et al., 1996). Others found no changes in cortex (Léna et al., 2005), an increase in NREM sleep in thalamus (Kékesi et al., 1997), in REM sleep in medulla (Kodama et al., 1998), and in waking in pedunculopontine tegmental nucleus (Kodama and Honda, 1999). These studies had low temporal resolution, with sampling rates of $1-5$ min (average duration of NREM and REM sleep episodes in rats is $\sim 3.6$ and 1.6 min) (Tang et al., 2007). Almost all studies used microdialysis, whose sensitivity to spontaneous synaptic release has been ques- 
A

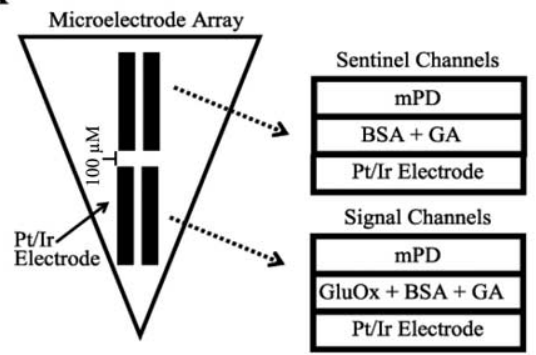

C

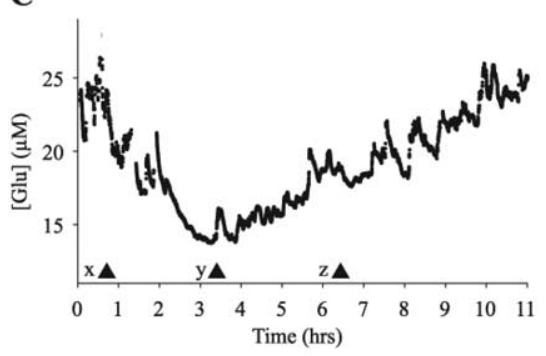

B

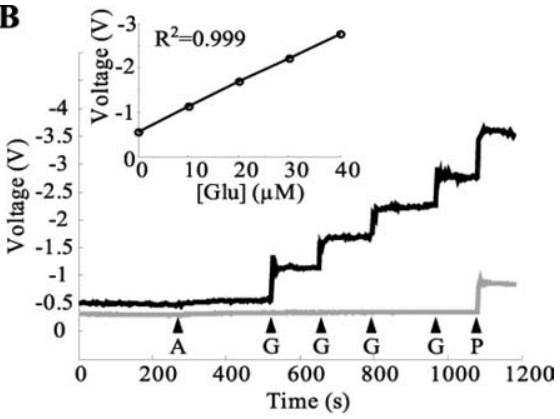

D

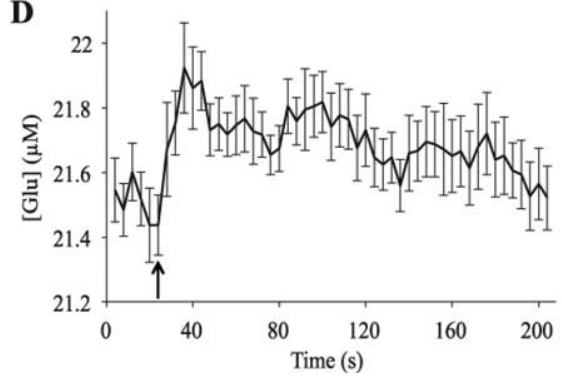

Figure 1. In vitro calibration and in vivo assessment of microelectrode arrays. $A$, Schematic of the self-referencing microelectrode arrays. Signal channels are coated with Glu0x and are responsive to glutamate, whereas sentinel channels are coated only with the cross-linking proteins BSA and glutaraldehyde (GA). Signal and sentinel channels both have an $m$-phenylenediamine exclusion layer (mPD). B, Typical in vitro calibration of signal (black line) and sentinel (gray line) channels. Arrows indicate the time of application of ascorbic acid (A;500 $\mu \mathrm{m})$, glutamate $(\mathrm{G} ; 10 \mu \mathrm{m})$, and peroxide (P). Both channels show little or no response to ascorbic acid but respond robustly to peroxide, whereas only the signal channel responds robustly to glutamate. Inset, Calibration curve demonstrating the linear response of the signal channels to changes in glutamate concentration. $C$, Changes in glutamate concentration after pentobarbital injection in one rat $(x ; 50 \mathrm{mg} / \mathrm{kg}$, i.p.). The first $90 \mathrm{~s}$ episode of arousal with locomotion was observed at $(y)$, but full motor activity was regained only at $(z)$. $D$, Rapid increase in glutamate concentration during whisker stimulation contralateral to an electrode implanted in the barrel cortex in one rat. The rat received 15 stimulations applied over the course of $90 \mathrm{~min}$. The average of all 15 stimulations, time locked to stimulation onset, is depicted in $4 \mathrm{~s}$ intervals. Stimulation onset began at arrow and each stimulation lasted on average $23.72 \pm 3.28 \mathrm{~s}$.

tioned (Timmerman and Westerink, 1997). Also, samples were collected for at most 2-6 h during the light period, and without resorting to sleep deprivation, so the effects of waking duration and sleep pressure on glutamate levels could not be assessed.

Here we use in vivo amperometry, which is sensitive to spontaneous synaptic release and has a temporal resolution of $<1 \mathrm{~s}$ (Burmeister et al., 2000; Rutherford et al., 2007), to measure changes in glutamate levels across the sleep-waking cycle in two cortical areas. Data were continuously collected for up to $4 \mathrm{~d}$ in baseline conditions and after sleep deprivation. We find that glutamate concentration increases rapidly and progressively during wakefulness and REM sleep, and decreases during NREM sleep, compensating for previous increases. Thus, the concentration of extracellular glutamate is kept within a homeostatic range across sleep-waking states.

\section{Materials and Methods}

Surgery. Male Wistar Kyoto rats ( $n=10$; Charles River), 300-350 g at time of surgery, were used in the present study. One week before surgery animals were individually housed in their recording chambers under controlled conditions $\left(24 \pm 1^{\circ} \mathrm{C} ; 12 \mathrm{~h}\right.$ light/dark cycle lights on at 10:00 A.M.) with access to food and water ad libitum. The recording chamber consisted of a clear Plexiglas enclosure $(36.5 \times 25 \times 46 \mathrm{~cm})$ in a soundattenuating box.

Under isoflurane anesthesia (3\% induction; $1.5 \%$ maintenance) local field potential (LFP) electrodes (Rhodes Medical Instruments) were implanted in the left parietal cortex (millimeters from bregma: anteroposterior: AP, -3.5 ; mediolateral: $\mathrm{ML},-3$ ) and right motor cortex ( $\mathrm{AP},+2$; $\mathrm{ML},+3)$. Two gold-plated epidural screws affixed to the scalp, one frontal (AP, +4; ML, -2) and one cerebellar (AP, -9; ML, 0), served as EEG electrodes. Silver wire electrodes were implanted in the nuchal muscles to monitor the electromyogram (EMG). Microelectrode arrays (MEAs; see below), were implanted in either motor cortex $(n=3$; AP, +2 ; ML, -3 ; dorsoventral: $\mathrm{DV},-1.5)$ or prefrontal cortex $(n=5$; $\mathrm{AP},+3.2 ; \mathrm{ML},-0.8 ; \mathrm{DV},-4.5)$, with a $\mathrm{Ag} / \mathrm{AgCl}$ reference electrode placed occipitally (AP, -6.5 ; $\mathrm{ML},+3)$. Two additional rats were also used in pilot studies (anesthesia and whisker stimulation, see below). For these studies, a MEA was implanted in motor cortex in one rat and barrel cortex (AP, -2.3 ; ML, -3.8 ; DV , -2) in the other. Dental cement was used to affix the electrodes to the animal's skull. Recordings began immediately after surgery. All animal procedures followed the National Institutes of Health Guide for the Care and Use of Laboratory Animals and facilities were reviewed and approved by the Institutional Animal Care and Use Committee of the University of Wisconsin-Madison and were inspected and accredited by the Association for Assessment and Accreditation of Laboratory Animal Care.

Recordings of behavioral states and sleep staging. EEG and EMG recording signals were connected to the commutator with a flexible cable. The signals were then sent to a polygraph (Grass Technologies, model 8-16 C), amplified and filtered (EEG and LFP signals: high-pass at $0.1 \mathrm{~Hz}$, low-pass at $70 \mathrm{~Hz}$; EMG signals: high-pass at 5 $\mathrm{Hz}$, low-pass at $70 \mathrm{~Hz}$ ). All signals were notchfiltered at $60 \mathrm{~Hz}$. Analog signals were digitized with the analog-to-digital (A/D) board (National Instruments) and sampled at $128 \mathrm{~Hz}$ (VitalRecorder, Kissei Comtec). Vigilance states were determined off-line by visual inspection of EEG and EMG signals and scored in 4 s epochs as either awake (low-voltage, high-frequency EEG, high EMG), NREM sleep (high-voltage, low-frequency EEG, low EMG), or REM sleep (lowvoltage, high-frequency EEG, low EMG activity). Vigilance state could be determined for all epochs.

$\mathrm{Ag} / \mathrm{AgCl}$ reference electrode preparation. $\mathrm{Ag} / \mathrm{AgCl}$ reference electrodes were prepared as previously described (Rutherford et al., 2007). Briefly, a Teflon-coated silver wire (Cooner Wire) was stripped at both ends $(0.25$ inch). A gold-plated socket (Ginder Scientific) was soldered to one end and connected to the cathode of a 9V DC current power supply. A second silver wire was stripped and attached to the anode of the power supply. These wires were immersed in $1 \mathrm{M} \mathrm{HCl}$ saturated with $\mathrm{NaCl}$ and current was applied for $15 \mathrm{~min}$ to coat the reference electrode with $\mathrm{AgCl}$.

Microelectrode preparation. Ceramic-based MEAs (Center for Microelectrode Technology) containing four, $15 \times 333 \mu \mathrm{M}$ platinum/iridium electrodes were modified for chronic implantation in freely moving rats as previously described (Rutherford et al., 2007). Implantable assemblies were cleaned by submersion in three baths ( 5 min Citrisolv, $5 \mathrm{~min}$ isopropyl alcohol, 5 min DI water; all reagents from Sigma) and dried for 15 min in an $80^{\circ} \mathrm{C}$ oven. After cleaning, a coating solution of L-glutamate oxidase (GluOx; Associates of Cape Cod Incorporated), glutaraldehyde (Sigma), and bovine serum albumin (BSA; Sigma) was applied to two recording channels. GluOx catalyzes the conversion of glutamate to $\alpha$-ketoglutarate and hydrogen peroxide. With an applied potential, $\mathrm{H}_{2} \mathrm{O}_{2}$ can then oxidize at the surface of the recording channels and acts as a reporter molecule for glutamate. Glutaraldehyde and BSA were included to affix the GluOx to the recording channels. The remaining two recording channels were coated with only glutaraldehyde and BSA to serve as sentinel recording channels (Fig. 1A). These sentinel channels measure background current from possible interferents only. Signal and sentinel channels are in close proximity in the microelectrode array, with a distance of $100 \mu \mathrm{m}$ separating the edge of the signal channels from the edge 
of the sentinel channels (Fig. 1A). Subtracting current sensed by sentinel channels from current sensed by signal channels provides a signal reflecting glutamate concentration, removes background current produced by other analytes and reduces noise (Burmeister and Gerhardt, 2001; Rutherford et al., 2007). After application of the coatings, the MEAs were allowed to dry overnight at $4^{\circ} \mathrm{C}$. The following day the MEAs were submerged in a $5 \mathrm{~mm}$ m-phenylenediamine (MPD; Sigma) solution of $0.05 \mathrm{M}$ phosphate-buffered solution (PBS). Cyclic amperometry (Peak-Peak: 0.25 , offset: -0.5 , frequency 0.05 ) was applied for $30 \mathrm{~min}$ to electroplate the MEA with a MPD exclusion layer. This size-exclusion layer serves to limit interference in vivo from electroactive analytes such as ascorbate without disrupting detection of the analyte of interest, glutamate.

Electrode calibration, in vivo amperometry, and postprocessing. Microelectrodes were calibrated in vitro before implantation in freely moving animals. The microelectrodes were submerged in $40 \mathrm{ml}$ of $0.05 \mathrm{M} \mathrm{PBS}, \mathrm{pH}$ 7.4 , which was stirred and maintained at $37^{\circ} \mathrm{C}$ by means of a water bath (Gaymar). Fixed-potential amperometry $(+0.7 \mathrm{~V}$ vs Ag/AgCl reference) was applied to recording electrodes using the FAST-16 potentiostat (Fast Analytical Sensing Technology). Responses of both signal and sentinel channels to ascorbic acid (final concentration, $250 \mu \mathrm{M}$; Sigma), monosodium glutamate (four sequential additions of $10 \mu \mathrm{m}$ increments; Sigma), and hydrogen peroxide were recorded and stored off-line.

For in vivo recordings, fixed-potential amperometry $(+0.7 \mathrm{~V}$ vs Ag/ $\mathrm{AgCl}$ reference) was performed using a four channel potentiostat (FAST16). The applied potential of $+0.7 \mathrm{~V}$ was chosen to enable oxidization of hydrogen peroxide (the reporter molecule for glutamate) while minimizing interference from other analytes that could otherwise oxidize at a higher applied potential. Implantable MEAs were connected to a headstage amplifier (500X, Rat Hat; Quanteon) to amplify current produced from oxidation at the surface of the electrodes. The headstage amplifier was connected with a flexible cable to a commutator (Airflyte) to enable freedom of movement within the recording apparatus. The commutator was connected to the potentiostat where the signal was furthered amplified $(10 \times)$. Analog outputs from the potentiostat were digitized with an A/D board (National Instruments) and recorded to hard drive with a sampling rate of $128 \mathrm{~Hz}$ (VitalRecorder, Kissei Comtec). In vivo recordings began immediately after surgery and lasted for up to $4 \mathrm{~d}$ postsurgery. In one pilot animal, pentobarbital anesthesia $(50 \mathrm{mg} / \mathrm{kg}$, i.p.) was administered $1 \mathrm{~h}$ after light onset on postsurgery day 2 while the concentration of glutamate was recorded in motor cortex. After loss of righting reflex, the animal was placed on an electric heating pad to maintain proper body temperature. Upon full behavioral recovery, the heating pad was removed and recordings continued uninterrupted. In a second pilot animal, whisker stimulation was performed by gently deflecting whiskers contralateral to the implanted MEA with a small paintbrush while the extrasynaptic concentration of glutamate in barrel cortex was assessed. The rat remained awake throughout the course of the stimulations that were administered over the course of $90 \mathrm{~min}$.

As previously described (Rutherford et al., 2007), the absolute strength of the glutamate signal recorded from signal channels declined over time, likely as a result of degradation or fouling of the applied enzyme. In our experiments, this was evident as a slow, but progressive decay in total current sensed by the signal channels across several days. To account for this decay and facilitate comparison between experimental days, the linear component of this decay was removed before analysis of glutamate concentrations. Examples of the removal of linear decay can be seen in supplemental Figure 1, available at www.jneurosci.org as supplemental material.

Signals from microelectrode recordings were processed with Matlab (Mathworks). Each signal was low-pass filtered (chebyshev type II, passband $=18 \mathrm{~Hz}$, stopband $=19 \mathrm{~Hz}$ ). The concentration of glutamate was calculated by dividing the raw voltage recorded during the experiment by the sensitivity of individual electrodes (volts/ $\mu \mathrm{M}$ glutamate) determined from previous in vitro calibration of each individual electrode. Final glutamate concentration was obtained by subtracting sentinel channels from signal channels. To measure changes in glutamate concentration based on behavioral state, the concentration of glutamate was binned and averaged over $4 \mathrm{~s}$ epochs previously scored as waking, NREM, or REM sleep. To remove technical artifacts present in some recordings, a $5 \mathrm{~min}$ moving median filter was applied to each signal and values that deviated from this median by $\pm 40 \%$ were removed. This procedure reliably removed large technical artifacts and for each chronic recording removed no $>5 \%$ of data points. Statistical tests were performed using Matlab and Statistica (Statsoft).

Histology. At the end of the experiment rats were deeply anesthetized with isoflurane anesthesia ( $3 \%$ in oxygen) and perfused transcardially with saline immediately followed by $4 \%$ solution of paraformaldehyde (PFA) in $0.1 \mathrm{M}$ sodium phosphate, $\mathrm{pH}$ 7.2. Brains were postfixed overnight in $4 \%$ PFA, cryoprotected in increasing concentration of sucrose $(15,20,30 \%)$ in PBS, and rapidly frozen on dry ice. Brains were then cut into $40 \mu \mathrm{m}$ serial coronal sections, mounted, and stained with cresyl violet. Placement of glutamate and LFP electrodes was determined using light microscopy. In all cases electrodes were in the targeted cortical regions and did not extend to the white matter below cortical layer VI. No excessive or unexpected tissue damage was noted.

\section{Results \\ In vitro calibration shows the specificity of the glutamate signal}

Each individual electrode was calibrated before implantation. Figure 1 shows a typical calibration experiment, which assessed the responsiveness of a glutamate-sensitive signal channel as well as a glutamate-insensitive sentinel channel (see Materials and Methods for details). Across all calibrations, signal channels ( $n=$ $20)$ were highly sensitive to changes in glutamate concentrations $(0.048 \pm 0.006 \mathrm{~V} / \mu \mathrm{M})$ and displayed limits of detection in the low micromolar range $(0.845 \pm 0.154 \mu \mathrm{M})$. Signal channels responded linearly to changes in glutamate concentrations, demonstrating that changes in output voltage reflect changes in glutamate concentration. Sentinel channels, instead, were unresponsive to glutamate.

Ascorbic acid and hydrogen peroxide challenges were administered to test the selectivity of the electrodes and ensure that signal and sentinel channels differed only in their responsiveness to glutamate (Fig. $1 B$ ). The MPD exclusion layer applied to the surface of the electrodes limited the response of the electrodes to interference from ascorbic acid without affecting either the signal or sentinel channels' robust responses to a hydrogen peroxide challenge. For subsequent in vivo recordings, electrodes were only used if the selectivity to glutamate over ascorbic acid was $>50: 1$, and if the response to the hydrogen peroxide challenge did not differ by $>15 \%$ in the signal relative to the sentinel channels.

\section{Cortical glutamate concentrations reflect increases and decreases in synaptic activity}

Glutamate levels as measured by amperometry are sensitive to block of action potentials by tetrodotoxin (Day et al., 2006; Oldenziel et al., 2006), whereas this treatment does not consistently affect the concentration of glutamate as measured with microdialysis (Timmerman and Westerink, 1997). Thus, this method may reflect glutamate released from synapses better than microdialysis (Timmerman and Westerink, 1997; Baker et al., 2002; van der Zeyden et al., 2007). To further assess whether the microelectrodes we used are sensitive to changes in glutamate concentration in vivo, pentobarbital (50 mg/kg, i.p.), an anesthetic known to augment inhibition, decrease neuronal activity, and depress excitatory neurotransmission (Nicoll and Madison, 1982; Richards and Strupinski, 1986; Wan et al., 2003), was administered to one rat while recording the concentration of glutamate in motor cortex (Fig. 1C). As expected pentobarbital administration produced immobilization, loss of righting reflex, and, within $30 \mathrm{~min}$ from the injection, an EEG pattern dominated by slow waves that persisted for $\sim 2 \mathrm{~h}$. During this time, the concentration of gluta- 
Table 1. Sleep parameters (mean \pm SEM, $n=8$ rats) during two baseline days

\begin{tabular}{lrrr}
\hline & Daily total $(\mathrm{h})$ & Light (\%) & Dark (\%) \\
\hline Wake & $9.63(0.39)$ & $29.29(1.61)$ & $51.71(1.86)$ \\
NREM & $12.48(0.35)$ & $60.72(1.30)$ & $44.05(1.76)$ \\
REM & $1.70(0.13)$ & $9.96(0.71)$ & $3.47(0.13)$ \\
\hline
\end{tabular}

Because day 1 and day 2 were not significantly different, data were pooled.

mate progressively decreased and reached a nadir of $14.01 \mu \mathrm{M}$; a $42 \%$ decrease from its initial concentration of $24.26 \mu \mathrm{M}$. The first brief arousal was observed $2.5 \mathrm{~h}$ after administration, when the rat was mobile for $\sim 90 \mathrm{~s}$ and the EEG showed a high frequency low amplitude activated pattern similar to that of normal waking. The animal then remained immobile for 3 more hours before full motor activity was regained, $5.5 \mathrm{~h}$ after the initial anesthetic injection. Glutamate concentrations began to increase immediately during the $90 \mathrm{~s}$ episode of arousal, and continued to rise afterward up to a concentration of $18.69 \mu \mathrm{M}$ immediately before full recovery of motor activity. Glutamate returned to prepentobarbital levels $8.5 \mathrm{~h}$ after the initial administration. Hence, pentobarbital, known to depress synaptic activity, produced a large decrease in the extrasynaptic concentration of glutamate as recorded by our microelectrodes. These decreases are consistent with published data under urethane anesthesia (Rutherford et al., 2007).

The experiment under pentobarbital anesthesia demonstrates that a decrease in the concentration of glutamate can be observed after a treatment known to depress synaptic activity. To investigate whether the opposite is true, namely that an increase in the concentration of glutamate can be observed after an increase in synaptic activity, another pilot experiment was undertaken in which the effects of whisker stimulation on the concentration of glutamate in barrel cortex were assessed. Whisker stimulation activates neurons in the contralateral barrel cortex, producing a complex pattern of spikes that are mediated by glutamatergic transmission (Armstrong-James et al., 1993). A single rat was subjected to 15 whisker stimulations (each lasting 20 s, 3-4 min apart), which reliably produced an increase in the extrasynaptic concentration of glutamate in the contralateral barrel cortex (Fig. $1 D)$. Specifically, we observed a rapid increase in the concentration of glutamate that persisted throughout the duration of each stimulation. The concentration of glutamate at the conclusion of each stimulation was always elevated compared with prestimulation levels. On average, glutamate concentrations increased by $0.459 \pm 0.08 \mu \mathrm{M}$ during whisker stimulations (average stimulus duration: $23.72 \pm 3.28 \mathrm{~s}$ ), consistent with previous reports (Pomerleau et al., 2003). In the same rat, no increase was observed during stimulation of whiskers ipsilateral to the microelectrode $(n=7)$ (supplemental Fig. 2, available at www.jneurosci.org as supplemental material). Thus, the microelectrodes in the present study are sensitive to bidirectional changes in the concentration of glutamate after either an increase or a decrease in synaptic activity.

\section{Sleep-wake parameters}

Simultaneous polysomnographic and amperometric recordings were performed continuously in freely behaving animals. In previous experiments polygraphic recordings started at least 1 week after surgery, to allow full recovery. In the current experiments, however, due to the progressive decay in the absolute strength of the glutamate signal (see Materials and Methods), all rats were recorded starting $24 \mathrm{~h}$ after the surgery. Recordings lasted at least 2 full days, and in some cases up to $4 \mathrm{~d}$.
Table 1 shows sleep quantity and quality for the 2 baseline recording days (postsurgery day 2 and 3 ). Since there was no significant difference between the $2 \mathrm{~d}$ in any of the sleep parameters investigated the data were pooled. Rats spent $\sim 60 \%$ of the $24 \mathrm{~h}$ period asleep, compared with $\sim 50 \%$ in fully recovered rats (Alföldi et al., 1991), but they still were more awake at night (52\% of the time) than during the day (29\%). The total increase in sleep was mainly due to more time spent in NREM sleep at night (NREM, 61 and 44\% in light and dark period, respectively, vs 55 and $25 \%$ in fully recovered rats; REM sleep, 10 and $4 \%$ vs 12 and $6 \%$ in fully recovered rats) (Alföldi et al., 1991). The average duration of NREM and REM sleep episodes (episode criteria: minimum duration, $16 \mathrm{~s}$; maximum allowed interruption, $16 \mathrm{~s}$ ) was $3.0 \pm 0.2 \mathrm{~min}$ and $1.54 \pm 0.1 \mathrm{~min}$, respectively, similar to those reported for rats on day 2 and 3 after surgery (Tang et al., 2007). Waking activities such as grooming, exploration, eating and drinking did not show obvious abnormalities although subjects typically appeared less active than fully recovered rats.

\section{Cortical glutamate concentrations reflect changes in sleep and waking independent of time of day}

Chronic amperometry was used to assess changes in the extracellular concentration of glutamate across the sleep-waking cycle in motor cortex or prefrontal cortex. Across the entire $2 \mathrm{~d}$ baseline period the average concentration of glutamate was $18.85 \pm 6.4$ $\mu \mathrm{M}$ in motor cortex $(n=3)$ and $15.27 \pm 4.1 \mu \mathrm{M}$ in prefrontal cortex $(n=5)$. Since results were not significantly different between the two areas $\left(t_{(6)}=0.50, p=0.64\right.$; independent samples, two-tailed), the data were pooled. The average concentration of glutamate across all rats was $16.6 \pm 3.3 \mu \mathrm{M}$, a value within the range previously reported in the cerebral cortex of other strains of rats (Rutherford et al., 2007) and in mice (Hascup et al., 2008). As in previous studies, we also found significant interindividual variability. Specifically, average glutamate concentrations ranged from as low as $6-8 \mu \mathrm{M}$ in a few animals to as high as $26-30 \mu \mathrm{M}$ in others. Of note, the concentration of glutamate sampled with amperometry typically (including the present study) exceeds the range of concentrations reported by microdialysis studies (1-3 $\mu \mathrm{M}$ ) (van der Zeyden et al., 2007), most likely due to closer proximity of microelectrodes to synapses, higher sensitivity of amperometry recordings to neuronal activity, and higher sampling rates (Rutherford et al., 2007).

Despite the variability in absolute values, in all rats the concentration of glutamate was not static, but varied throughout the recording period. This is shown in Figure 2, where absolute glutamate levels during a $24 \mathrm{~h}$ baseline period are plotted for each consecutive $4 \mathrm{~s}$ epoch scored as waking or sleep. The rat in Figure $2 A$ (each panel refers to a different animal) was well entrained to the light/dark cycle and, as typically seen in fully recovered rats, was mainly asleep during the day and showed long periods (as long as $58.5 \mathrm{~min}$ ) of continuous waking during the night. It is clear that glutamate levels increase for as long as the rat is awake, and decrease or stay low during sleep. The rat in Figure $2 B$ was also mainly awake during the night and asleep during the day, but showed a more fragmented sleep pattern, with episodes of con- 
tinuous waking no longer than $20 \mathrm{~min}$ : in this rat glutamate levels also increased throughout waking, and decreased during sleep. The rat in Figure $2 C$ instead slept significantly also during the night. In this rat glutamate levels kept decreasing for most of the light period and the first hours of the dark period, and then remained low. Finally, Figure $2 D$ refers to a rat that showed long periods of waking also at the beginning of the light period: glutamate levels increased during this time as long as the rat was awake, and reached peak values as high as at the end of long waking bouts during the night. Overall, these data show that in the rat prefrontal and motor cortex glutamate levels (1) increase during waking; (2) decrease or remain low during sleep; (3) are not affected by the transition from light to dark, per se; (4) are not affected by time of day independent of behavioral state.

\section{Cortical glutamate levels increase progressively during both waking and REM sleep, and decrease progressively in NREM sleep}

Since NREM sleep and REM sleep differ sig-

nificantly in terms of metabolism and neuronal activity, we examined whether they also have different effects on cortical glutamate levels. Figure 3 shows absolute glutamate levels plotted for each consecutive $4 \mathrm{~s}$ epoch in 2 rats, but unlike in Figure 2, NREM sleep and REM sleep are scored separately.

It is clear that both in prefrontal and motor cortex glutamate levels increase not only during waking, but also during REM sleep. Moreover, as evident from Figures 2 and 3, changes in concentration were not confined to the transitions between behavioral states, but occurred progressively throughout spontaneous waking, NREM, and REM periods. To quantify these changes over time, we considered all consolidated periods of sleep or waking during the 2 baseline days, defined as those episodes whose duration was equal or greater than the mean duration for each behavioral state $(2.5,3$, and 1.5 min for waking, NREM, or REM sleep, respectively). These criteria enabled the characterization of a large number of episodes (mean \pm SEM in $24 \mathrm{~h} / \mathrm{rat}$ : waking, $43.5 \pm 4.1$; NREM, $91.9 \pm 4.0$; REM, $24.0 \pm 2.4)$ that were sufficiently long to minimize technical variability in assessing glutamate levels, which is more pronounced for short episodes containing only a few 4 s epochs. For each rat, we calculated the percentage change in glutamate concentration for each consolidated episode of sleep or waking relative to each rat's overall $2 \mathrm{~d}$ mean concentration (across all behavioral states), and averaged them to provide an index of the typical changes in glutamate concentration for waking, NREM, and REM sleep. As can be seen in Figure 4, the concentration of glutamate progressively increased during the vast majority of waking and REM episodes and progressively decreased during most NREM episodes. Within each behavioral state, the average changes that were observed in prefrontal cortex did not significantly differ from those observed in motor cortex (waking: $t_{(6)}=0.073, p=0.944$; NREM: $t_{(6)}=$ $1.37, p=0.221$; REM: $\left.t_{(6)}=0.663 p=0.532\right)$. Consequently, data from these two regions were pooled for further analyses. Across all rats, glutamate concentrations increased $0.329 \pm 0.06 \% / \mathrm{min}$ during waking and $0.349 \pm 0.13 \% / \mathrm{min}$ during REM sleep, and
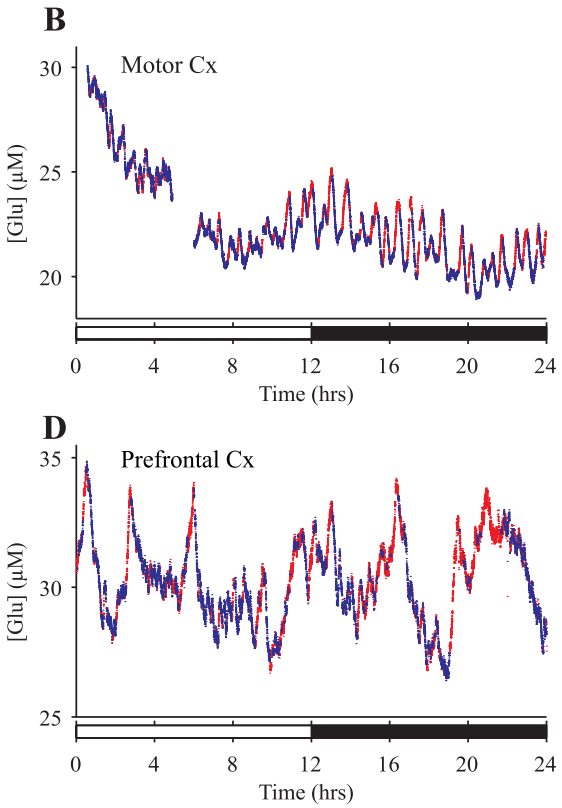
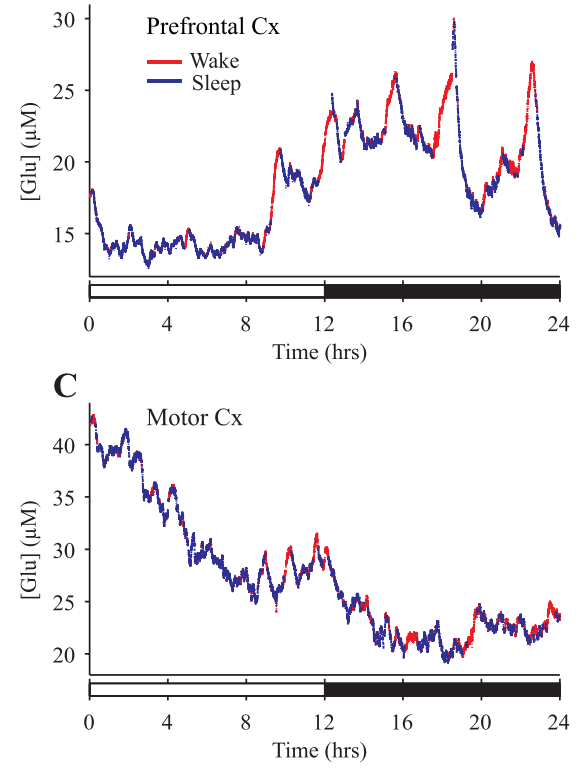

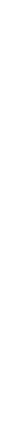
during sleep and waking across the light/dark period for four individual rats. Each data point represents the average concentration of glutamate across a $4 \mathrm{~s}$ window of waking (red) or sleep (blue). Absolute glutamate concentrations are highly variable across the light/dark period but display consistent changes within behavioral states. Cx, Cortex.

decreased $0.338 \pm 0.06 \% / \mathrm{min}$ during NREM sleep. Changes in glutamate concentration during waking or REM sleep were significantly different from changes during NREM (repeated measure ANOVA, $F_{(2,14)}=16.94, p<0.001$; post hoc Tukey's HSD, $p<0.001$ ), whereas no significant difference was observed between waking and REM. It should be pointed out that these results, based on mean episode duration, did not significantly change when glutamate levels were calculated across a wide range of episode length (1-5 min for waking and NREM, 0.5-3 min for REM), or when the median episode duration for each behavioral state was considered.

There were a few episodes during which glutamate concentrations exhibited atypical changes for a given behavioral state as shown in Figure 4. Specifically, a small minority (6.6\%) of all consolidated NREM periods showed the opposite trend, i.e., a progressive increase in glutamate concentration during NREM sleep that was at least as large as the average decrease normally observed during this stage of sleep. We could not find an obvious explanation for the opposite behavior of glutamate in these NREM episodes, as factors such as the occurrence of a REM episode or a brief awakening immediately afterward did not seem to matter. However, as shown in Figure 4, a subset (12.3\%) of consolidated waking periods also showed the opposite trend, i.e., the concentration of glutamate progressively decreased, and the reduction over time was as significant as the increase normally seen during waking. The waking episodes with decreasing glutamate levels were more often $(60 \%$ of cases) interspersed within long and consolidated NREM periods (at least 85\% of NREM sleep during a $>10 \mathrm{~min}$ recording period), which are typical of sleep under high sleep pressure (i.e., sleep at the beginning of the major sleep phase, and sleep after sleep deprivation). Hence, glutamate concentrations exhibit a progressive rise during spontaneous waking with the exception of some short periods of waking interspersed within NREM episodes during high sleep pressure. Finally, as in waking, in a subset $(15.5 \%)$ of REM epochs glutamate concentrations showed a decrease rather than an increase. 
A

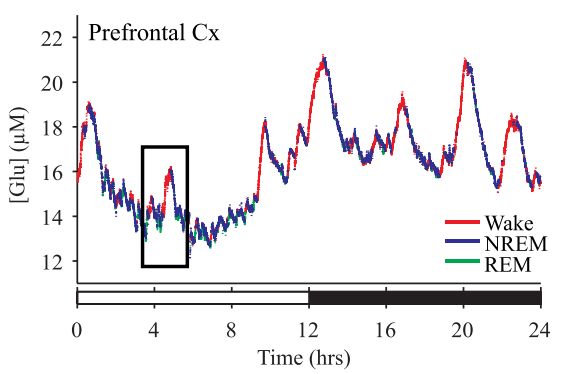

$$
\text { C }
$$

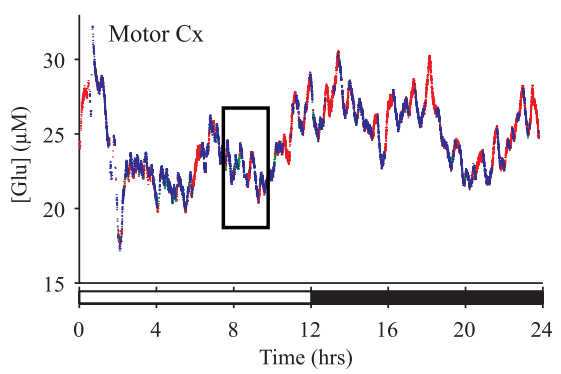

B

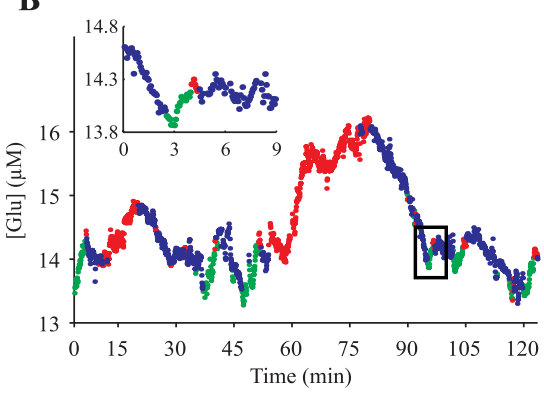

D

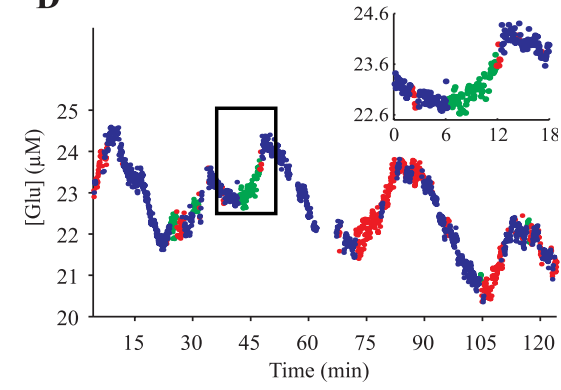

Figure 3. Dynamic changes in extrasynaptic glutamate concentration during waking, NREM, and REM sleep. $\boldsymbol{A}$ shows a $24 \mathrm{~h}$ recording in the prefrontal cortex ( $(\mathrm{x})$ of a single rat. Each data point represents the average concentration of glutamate across a 4 s window of waking (red), NREM (blue), or REM (green). The $2 \mathrm{~h}$ window boxed in $\boldsymbol{A}$ is depicted at higher resolution in $\boldsymbol{B}$, with the inset showing a single NREM-to-REM transition. $C$ and $\boldsymbol{D}$ are organized as $\boldsymbol{A}$ and $\boldsymbol{B}$, but show $24 \mathrm{~h}$ recording in the motor cortex of a different rat.

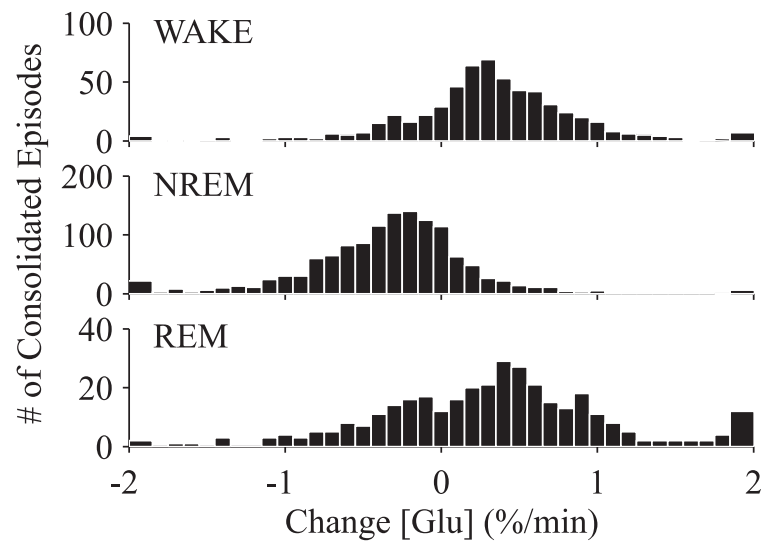

Figure 4. Behavioral-state-dependent changes in glutamate concentration. Histogram of percentage change in glutamate concentration during consolidated periods of waking (all episodes $>2.5 \mathrm{~min})$, NREM ( $>3 \mathrm{~min})$, or REM (>1.5 $\mathrm{min})$ for all rats $(n=8)$. The observed changes in prefrontal and motor cortex were not significantly different from one another for any behavioral state, and thus data were pooled ( $n=8$ ). Values outside of $x$-axis scale (those with $>2 \% / \mathrm{min}$ ) were grouped in the edge bins.

We do not have a clear explanation for these atypical epochs, but it is likely that at least some of these apparent changes in the opposite direction reflect higher technical variability, due to the shorter duration of REM bouts.

As mentioned before, recordings lasted for up to $4 \mathrm{~d}$ starting $24 \mathrm{~h}$ after the surgery. Thus, the changes in glutamate described above during waking, NREM, and REM may have been influenced by the close proximity to surgery, since our rats spent more time asleep than fully recovered animals, especially at night. To determine whether this was the case we ran 2 sets of analyses. First, we focused on the fourth day postsurgery in a single animal in which the strength of the glutamate signal remained high throughout that day. This rat displayed a sleep pattern more sim- ilar to that seen in fully recovered animals (percentage of $24 \mathrm{~h}$ : waking, 53.8\%; NREM, 43.1\%; REM, 3.0\%; percentage of dark period: NREM, 32.0\%; REM, 3.2\%), and yet, the changes in glutamate concentration during this day were not significantly different from those observed in the same animal on day 1 and day 2 postsurgery ( $p>0.05$ for waking, NREM and REM). In a second analysis we selected, among all baseline recording days, those that showed sleep patterns the most $(n=3)$ or the least $(n=3)$ similar to that of fully recovered rats (most similar, percentage of $24 \mathrm{~h}$ : waking, 49\%; NREM, 45\%; REM, 6\%; percentage of dark period: NREM, 34\%; REM, 4\%; least similar, percentage of 24 h: waking, 36\%; NREM, 58\%; REM, 6\%; percentage of dark period: NREM, 51\%; REM, 3\%). Again, there was no significant difference in the changes in glutamate within waking, NREM, or REM between the 2 groups. Thus, our results do not seem to be affected by the extent to which the sleep pattern was disrupted because of the surgery.

\section{Changes in glutamate levels during sleep deprivation}

The progressive increase in glutamate during most consolidated waking episodes raises the possibility that glutamate levels would continue to rise if waking was not interrupted by NREM sleep. To further characterize the effect of prolonged wakefulness on the time course of changes in glutamate, a subset of animals $(n=5)$ were deprived of sleep for $3 \mathrm{~h}$ by introducing novel objects to their cage to stimulate exploratory behavior. Pilot studies $(n=3)$ had indicated that despite the proximity to surgery (sleep deprivation started at light onset the fourth day after surgery) we could reliably maintain wakefulness with this method for the duration of the deprivation. After $3 \mathrm{~h}$ of sleep deprivation, rats were then allowed to sleep ad libitum.

Figure 5 depicts changes in glutamate concentration in the prefrontal cortex of 3 rats before, during, and after $3 \mathrm{~h}$ of sleep deprivation. During sleep deprivation glutamate concentrations initially rose in all 3 animals, consistent with the changes observed during spontaneous waking. Unlike during spontaneous waking, however, glutamate levels did not continue to rise for the entire duration of forced waking. The time when glutamate levels no longer increased varied among animals, being as short as 30 $\mathrm{min}$ in one rat (Fig. 5B) and as long as $120 \mathrm{~min}$ in another (Fig. $5 C$ ). Strikingly, across all 5 rats, the initial rise in glutamate continued only until the concentration reached a level similar to the highest concentration observed during spontaneous waking. Afterward, despite continued wakefulness as assessed by both behavioral and EEG analysis, glutamate concentrations either leveled (Fig. $5 B$ ), began to decrease (Fig. $5 C$ ), or first leveled and then decreased (Fig. 5A). Interestingly, the duration of the "increase" phase seems to depend on the previous sleep-waking history, so that the rat that had spent most of the last $2 \mathrm{~h}$ asleep (Fig. 5C) showed the longest increase, whereas the rat that was mostly awake for $1-2 \mathrm{~h}$ before the sleep deprivation began (Fig. $5 B)$ showed the shortest increase. Indeed, across all rats $(n=5)$, the amount of time spent in NREM sleep during the $90 \mathrm{~min}$ preceding the sleep deprivation was significantly correlated $(r=$ 
0.961, $p<0.01)$ with the duration of the increase phase (defined as the time required for glutamate levels to reach $95 \%$ of the peak value observed during sleep deprivation). Thus, glutamate concentrations increased for a longer time during sleep deprivation in those rats that were predominantly in NREM sleep before the start of sleep deprivation.

Figure $5 D$ shows the pooled data across the 5 animals: during the first hour of sleep deprivation the concentration of glutamate increased by $0.401 \pm 0.151 \% / \mathrm{min}$, an increase that was not significantly different from that observed during periods of spontaneous waking $\left(t_{(4)}=0.583, p=0.591\right.$; dependent samples $t$ test, two-tailed). During the second hour, however, the progressive increase in glutamate concentration slowed and flattened (0.133 $\pm 0.135 \% /$ $\mathrm{min}$ ), and during the third hour of deprivation glutamate concentration declined $(-0.430 \pm 0.106 \% / \mathrm{min})$, significantly different from spontaneous waking $\left(t_{(4)}=\right.$ 8.44, $p<0.01$; dependent samples $t$ test, two-tailed). Of note, there was a progressive increase in sleepiness throughout the sleep deprivation period, as indicated by an increase in the number of sleep attempts (defined as any period longer than $2 \mathrm{~s}$ of NREM sleep) as the deprivation progressed, especially between hour 1 and hours 2-3 (number of sleep attempts/h, mean \pm SEM, hour $1=4 \pm 0.95$; hour $2=$ $6.8 \pm 2.2$; hour $3=8.2 \pm 2.8$ )

During recovery sleep after sleep deprivation a large, progressive decrease in the concentration of glutamate was observed $(-0.710 \pm 0.131 \% / \mathrm{min}$ for the first $2 \mathrm{~h}$ after sleep deprivation). The rate of this decrease, occurring under the high sleep pressure that follows sleep deprivation, was significantly faster than the average rate of decline $(-0.338 \pm 0.06 \%$ / min) during spontaneous NREM sleep $t_{(4)}=4.48, p<0.05$; dependent samples $t$ test, two-tailed) suggesting again that intense sleep typical for high sleep pressure can modulate the changes in glutamate concentration associated with behavioral state. Moreover, glutamate levels at the end of recovery sleep reached values as low as, but not lower, than those observed after consolidated periods of baseline sleep during the light period. Overall, these data indicate that (1) glutamate concentrations rise during spontaneous and enforced wakefulness; (2) the increase in glutamate concentration during enforced waking does not persist indefinitely and reaches a peak concentration similar to that observed during spontaneous waking; (3) upon reaching this apparent threshold, glutamate concentrations plateau or decrease despite continued wakefulness and (4) glutamate concentrations decrease rapidly under high sleep pressure. rats ( \pm SEM, $n=5)$.
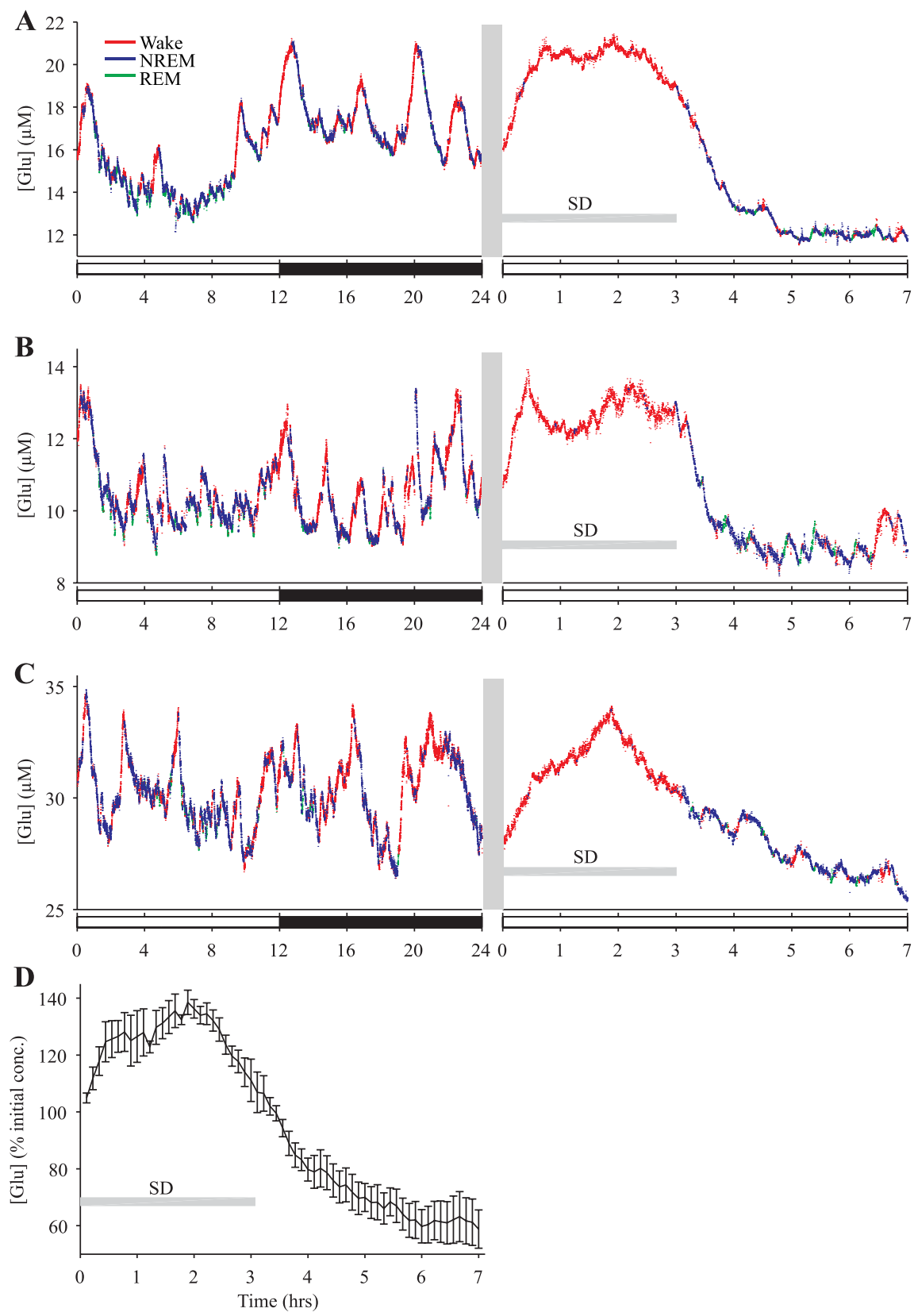

Figure 5. The effects of $3 \mathrm{~h}$ of sleep deprivation and subsequent recovery sleep on the concentration of glutamate in the prefrontal cortex. $\mathbf{A}$-C depict glutamate concentrations and behavioral state across a $24 \mathrm{~h}$ baseline day, $3 \mathrm{~h}$ of sleep deprivation, and $4 \mathrm{~h}$ of recovery sleep for three individual rats. Each data point represents the average concentration of glutamate across a $4 \mathrm{~s}$ epoch of waking (red), NREM (blue), or REM (green). Horizontal gray bars indicate sleep deprivation (SD). The vertical gray line indicates the $\sim 10$ min between the end of the baseline day (time $24 ; 10$ A.M.) and the onset of sleep deprivation (time 0 ) during which animal care was performed. $D$, Averaged response to $3 \mathrm{~h}$ of sleep deprivation and subsequent $4 \mathrm{~h}$ of recovery sleep for all

\section{Glutamate concentration and slow-wave activity}

The EEG pattern of NREM sleep is dominated by slow waves. Slow-wave activity (SWA) is defined as the EEG power in the $0.5-4 \mathrm{~Hz}$ band during NREM sleep, and is a reliable measure of the amplitude and number of slow waves during this phase of sleep. SWA is an established marker of sleep pressure, increasing with the duration of waking and dissipating throughout sleep (Achermann and Borbély, 2003). As expected, all rats showed the typical SWA profile across the light period, i.e., SWA was high immediately after sleep onset and declined progressively afterward (Fig. 6A). During the first $2 \mathrm{~h}$ of the light period, when sleep 

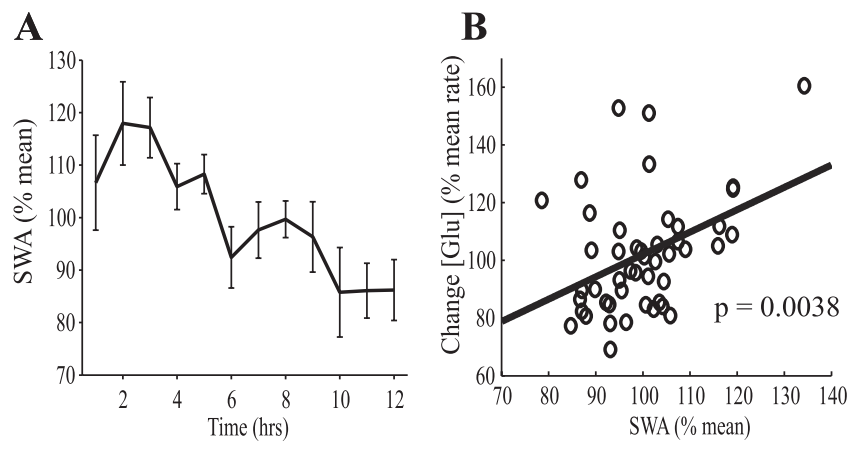

Figure 6. SWA and decreases in glutamate concentration during NREM sleep across the light period. $\boldsymbol{A}$, SWA exhibits a typical decline across the light period. $\boldsymbol{B}$, The rate of glutamate decline during NREM sleep was positively correlated $(r=0.41)$ with the amount of SWA. Each data point represents the average SWA and change in glutamate concentration during NREM sleep across $2 \mathrm{~h}$ blocks during the light period for each rat. One outlier ( $>3 \times \mathrm{SD}$ ) was removed, but the correlation remains significant even with its inclusion $(p=0.0496) .{ }^{*} p<0.05$.

pressure and SWA were high, the average rate of decrease in glutamate was also high $(-0.330 \pm 0.079 \% / \mathrm{min})$. Conversely, at the end of the major sleep phase (hour 9-10 of the light period), when sleep pressure and SWA were low, the average rate of decrease in glutamate levels during NREM sleep was also low $(-0.181 \pm 0.076 \% / \mathrm{min})$ and was significantly less than that observed during the first $2 \mathrm{~h}\left(t_{(7)}=2.92, p<0.05\right.$; dependent samples $t$ test, two-tailed). To further assess the relationship between sleep pressure and changes in extrasynaptic glutamate we also compared the rate of change in glutamate levels with the average SWA for each consolidated ( $>3 \mathrm{~min}$ ) NREM period during the light phase. All NREM sleep episodes were divided in quintiles based on average SWA levels, from the highest (top 20\% average SWA) to lowest (bottom 20\% average SWA). During NREM episodes with the highest SWA the average decrease in glutamate concentration was $-0.348 \pm 0.054 \% / \mathrm{min}$, significantly larger $\left(t_{(7)}=2.55, p<0.05\right.$; dependent samples $t$ test, two-tailed) than the decrease in glutamate concentration during NREM sleep episodes with the lowest SWA (bottom 20\% SWA; $-0.194 \pm 0.045 \% / \mathrm{min})$. Overall, we found that the rate of glutamate decline during NREM sleep and the levels of SWA were positively correlated $(r=0.41, p<0.01)$ (Fig. $6 B$ ). Thus, sleep pressure and sleep intensity as measured by SWA appear to change in parallel with the rate of decline in glutamate concentration during NREM sleep.

\section{Discussion}

As summarized in the introduction, several studies used microdialysis to assess glutamate levels across the sleep-waking cycle, but the results were inconsistent. Other studies used indirect markers to assess the effects of sleep and waking on glutamatergic signaling. Some reports measured tissue glutamate content in whole brain after total or REM sleep deprivation, with mixed results: no change (Karadzic et al., 1971; Himwich et al., 1973) or an increase in glutamate after sleep loss (Davis et al., 1969; Bettendorff et al., 1996). REM sleep deprivation was also shown to increase glutamine turnover (Mark et al., 1969), and protein levels of glutamine synthetase (Sallanon-Moulin et al., 1994). Transcript levels of genes coding for enzymes involved in glutamate synthesis (glutamine synthase, glutaminase), glutamate receptor subunits (GluR2, GluR3) (Cirelli and Tononi, 2000) and intracellular proteins implicated in glutamate receptor clustering (Homer/Vesl, Narp) are also upregulated during waking (Cirelli et al., 2004). Finally, the number of GluR1-containing AMPA receptors increases after waking relative to sleep in cortex and hippocampus (Vyazovskiy et al., 2008b). Thus, there is evidence that changes in glutamatergic signaling occur across behavioral states, but the link with glutamatergic neurotransmission is indirect.

Here we used in vivo amperometry to measure cortical extracellular glutamate continuously for several days with second-bysecond resolution. This allowed us to reach several novel conclusions. First, within each sleep-wake cycle $(<30 \mathrm{~min}$ in rats) glutamate levels increased rapidly and progressively during waking and REM sleep, and decreased progressively during NREM sleep. These dynamic within-state changes could not be detected with microdialysis due to its poor temporal resolution. Supporting our finding, a recent study focusing on the posterior hypothalamus used glutamate biosensors similar to those used here and found rapid increases in glutamate levels in waking and REM sleep during a $7 \mathrm{~h}$ window in the middle of the day (John et al., 2008).

Second, the minute-by-minute glutamate levels reflected the sleep-wake history of the previous several hours: glutamate levels were low in an awake rat if it had been asleep for several hours, and high in a sleeping rat if it had been mostly awake for the previous hours. Thus, the previous sleep-wake history contributes to the absolute levels of glutamate at any given time, whereas the direction of these changes is determined by the current behavioral state. Glutamatergic activity is associated with high energy demand (Attwell and Laughlin, 2001), and brain energy consumption is lower during NREM sleep relative to waking and REM sleep (Madsen et al., 1991; Maquet, 1995). Strikingly, cerebral metabolism in mice is also contingent upon the sleep-waking history (Vyazovskiy et al., 2008a). Specifically, the uptake of 2 -deoxyglucose during quiet waking is $15-20 \%$ lower in mice that had slept for $\sim 2.5 \mathrm{~h}$ before the injection of the tracer, compared with mice that had been awake before the injection (Vyazovskiy et al., 2008a).

Third, the sleep-waking history not only affected the absolute concentration of glutamate, but also modulated its rate of change. SWA is a reliable indicator of sleep pressure, and increases as a function of the duration of prior waking (Achermann and Borbély, 2003). Glutamate levels decreased at a faster rate during NREM sleep periods with high SWA than during those with low SWA. Overall, the rate of glutamate decline during NREM sleep was positively correlated with SWA. Also, the rate of decrease in glutamate during sleep after sleep deprivation, when sleep pressure is high, was approximately twice as fast as the average decrease during spontaneous NREM sleep. Hence, high sleep pressure enhances the NREM-related decline in glutamate levels.

Another factor modulating the changes in glutamate concentration during waking appears to be sleepiness. During sleep deprivation the frequency of sleep attempts increased, indicating an increase in sleepiness. Meanwhile, glutamate levels increased during the first hour of sleep deprivation, but by the third hour they started to decrease. Thus, toward the end of the deprivation, when rats attempted to sleep the most, the concentration of glutamate declined despite continued wakefulness. Changes in glutamate levels during spontaneous waking may also be affected by sleepiness, because most "atypical" waking epochs (associated with decreasing glutamate concentrations) occurred during short waking periods within long, consolidated NREM sleep periods.

The second-by-second concentration of glutamate in extrasynaptic space is the result of two opposite processes: extracellular release of glutamate from neurons and astrocytes, and intra- 
cellular uptake by high-affinity transporters. The fluctuations in extrasynaptic glutamate with behavioral state could therefore be due to changes in release or in transporter activity. Most glutamate uptake in cortex is mediated by the glutamate transporter-1, GLT-1 and the glutamate/aspartate transporter, GLAST (Tzingounis and Wadiche, 2007). Their expression/activity is modulated by many factors downstream of neuronal activity, including cAMP and kinases (Duan et al., 1999), and their block results in increased extracellular glutamate levels and neurodegeneration (Rothstein et al., 1996). The changes in glutamate levels observed in our study could be accounted for by decreased uptake during waking and REM sleep, and increased uptake during NREM sleep. There is no evidence, however, that GLT-1 and GLAST activity is reduced during waking. GLAST mRNA expression in rat cortex increases by $30 \%$ after $8 \mathrm{~h}$ of waking (Cirelli and Tononi, 2000), and GLAST expression increases after exposure to glutamate, resulting in increased uptake (Duan et al., 1999). Thus, if present, changes in glutamate transporter expression may serve to oppose the progressive increases in glutamate during waking and REM sleep, rather than contribute to it. The latter changes, instead, may reflect changes in glutamate release.

Under physiological conditions, glutamate is released into the extrasynaptic space by spillover from synapses (Asztely et al., 1997; Biró and Nusser, 2005; Sem'yanov, 2005), exocytosis from astrocytes (Santello and Volterra, 2008), and active transport by the cystine-glutamate antiporter (Baker et al., 2002). Extrasynaptic glutamate as measured by our microelectrodes is affected by block of action potentials by tetrodotoxin, indicating that it reflects neuronal activity (Day et al., 2006; Hascup et al., 2008). Astrocytic release of glutamate can be coupled with neuronal activity (Santello and Volterra, 2008), but the relative contribution of synaptic spillover and astrocytic release cannot be determined in our experimental conditions. Nevertheless, activityinduced release of glutamate is likely to underlie the changes in extrasynaptic glutamate concentration across the sleep-wake cycle.

Initial studies suggested that firing rates in cortex are higher during waking and REM sleep and lower during NREM sleep (Desiraju, 1972; Noda and Adey, 1973). Recent reports indicate that neuronal firing rates during NREM sleep are similar to waking during the up state of the slow oscillation, but are close to zero during the down state (Steriade et al., 2001). Therefore, the progressive rise of glutamate during waking and REM sleep could result from the inability of glutamate transporters to maintain a constant extrasynaptic glutamate concentration during high levels of neuronal activity. Meanwhile, during NREM sleep, the occurrence of down states could allow transporters to gradually reduce the extrasynaptic concentration of glutamate. Given that glutamate transporters complete a full cycle in $\sim 70 \mathrm{~ms}$ (Wadiche et al., 1995) and that down states typically last 80-300 ms (Ji and Wilson, 2007), each transporter could conceivably remove 1-4 molecules of glutamate per down state. Large slow waves, indicative of high sleep pressure, are associated with even longer silent periods of neuronal activity (V. Vyazovskiy, unpublished observations) (Calvet et al., 1973). This could account for the faster decline in the concentration of glutamate during NREM sleep with high SWA. It is tempting to speculate that NREM sleep may be a propitious time for replenishing presynaptic glutamate reserves depleted by tonic glutamate release during wakefulness, although there is so far little indication that the presynaptic glutamate pool may constitute a limited resource over long periods of time.

The progressive changes in extracellular glutamate reported here shows that, for whatever reason, glutamate release and clearance mechanisms are not kept in full balance in the short term. Instead, glutamate levels systematically increase during EEG activated states, raising the question whether they may become neurotoxic. In vitro studies of cultured neurons report glutamateinduced excitotoxicity at concentrations below those observed here (1-12 $\mu \mathrm{M})$ (Rosenberg et al., 1992; Cheung et al., 1998). However, astrocyte-rich cultures are much less sensitive to glutamate excitotoxicity $\left(\mathrm{EC}_{50}=205 \mu \mathrm{M}\right)$ (Rosenberg et al., 1992) $\left(\mathrm{EC}_{50}=100-200 \mu \mathrm{M}\right)$ (Regan and Choi, 1991), and high glutamate levels are required to produce excitotoxicity in vivo (Mangano and Schwarcz, 1983; Choi, 1988). Although it remains unclear whether the progressive increase of glutamate concentrations in waking can approach excitotoxic conditions, the present results show that NREM sleep effectively opposes glutamate accumulation in the extrasynaptic space. Thus, thanks to the alternation of wakefulness and NREM sleep, glutamate levels are kept within a homeostatic range, remaining relatively constant across the $24 \mathrm{~h}$ period. Moreover, as shown by the positive correlation between the rate of glutamate decline during NREM sleep and the levels of SWA, the homeostasis of extracellular glutamate in the cortex may be linked to sleep homeostasis. At this point, it is unknown whether NREM sleep is in any way critical to glutamate homeostasis, and it is likely that, in the absence of sleep, other compensatory mechanisms come into play. Perhaps the glutamate-decreasing effect of NREM sleep is especially relevant in pathological conditions, such as ischemia, that can lead to glutamate-induced excitotoxicity.

\section{References}

Achermann P, Borbély AA (2003) Mathematical models of sleep regulation. Front Biosci 8:s683-s693.

Alföldi P, Franken P, Tobler I, Borbély AA (1991) Short light-dark cycles influence sleep stages and EEG power spectra in the rat. Behav Brain Res 43:125-131.

Armstrong-James M, Welker E, Callahan CA (1993) The contribution of NMDA and non-NMDA receptors to fast and slow transmission of sensory information in the rat SI barrel cortex. J Neurosci 13:2149-2160.

Asztely F, Erdemli G, Kullmann DM (1997) Extrasynaptic glutamate spillover in the hippocampus: dependence on temperature and the role of active glutamate uptake. Neuron 18:281-293.

Attwell D, Laughlin SB (2001) An energy budget for signaling in the grey matter of the brain. J Cereb Blood Flow Metab 21:1133-1145.

Azuma S, Kodama T, Honda K, Inoué S (1996) State-dependent changes of extracellular glutamate in the medial preoptic area in freely behaving rats Neurosci Lett 214:179-182.

Baker DA, Xi ZX, Shen H, Swanson CJ, Kalivas PW (2002) The origin and neuronal function of in vivo nonsynaptic glutamate. J Neurosci 22:9134-9141.

Bettendorff L, Sallanon-Moulin M, Touret M, Wins P, Margineanu I, Schoffeniels E (1996) Paradoxical sleep deprivation increases the content of glutamate and glutamine in rat cerebral cortex. Sleep 19:65-71.

Biró AA, Nusser Z (2005) Synapse independence breaks down during highly synchronous network activity in the rat hippocampus. Eur J Neurosci 22:1257-1262.

Burmeister JJ, Gerhardt GA (2001) Self-referencing ceramic-based multisite microelectrodes for the detection and elimination of interferences from the measurement of L-glutamate and other analytes. Anal Chem 73:1037-1042.

Burmeister JJ, Moxon K, Gerhardt GA (2000) Ceramic-based multisite microelectrodes for electrochemical recordings. Anal Chem 72:187-192.

Calvet J, Fourment A, Thiefry M (1973) Electrical activity in neocortical projection and association areas during slow wave sleep. Brain Res 52:173-187.

Cheung NS, Pascoe CJ, Giardina SF, John CA, Beart PM (1998) Micromolar L-glutamate induces extensive apoptosis in an apoptotic-necrotic continuum of insult-dependent, excitotoxic injury in cultured cortical neurones. Neuropharmacology 37:1419-1429. 
Choi DW (1988) Glutamate neurotoxicity and diseases of the nervous system. Neuron 1:623-634.

Cirelli C, Tononi G (2000) Gene expression in the brain across the sleepwaking cycle. Brain Res 885:303-321.

Cirelli C, Gutierrez CM, Tononi G (2004) Extensive and divergent effects of sleep and wakefulness on brain gene expression. Neuron 41:35-43.

Davis JM, Himwich WA, Stout M (1969) Cerebral amino acids during deprivation of paradoxical sleep. Biol Psychiatry 1:387-390.

Day BK, Pomerleau F, Burmeister JJ, Huettl P, Gerhardt GA (2006) Microelectrode array studies of basal and potassium-evoked release of L-glutamate in the anesthetized rat brain. J Neurochem 96:1626-1635.

Desiraju T (1972) Discharge properties of neurons of the parietal association cortex during states of sleep and wakefulness in the monkey. Brain Res 47:69-75.

Duan S, Anderson CM, Stein BA, Swanson RA (1999) Glutamate induces rapid upregulation of astrocyte glutamate transport and cell-surface expression of GLAST. J Neurosci 19:10193-10200.

Hascup KN, Hascup ER, Pomerleau F, Huettl P, Gerhardt GA (2008) Second-by-second measures of L-glutamate in the prefrontal cortex and striatum of freely moving mice. J Pharmacol Exp Ther 324:725-731.

Himwich WA, Davis JM, Stout M (1973) Cerebral amino acids of rats deprived of paradoxical sleep. Biol Psychiatry 6:37-44.

Jasper HH, Khan RT, Elliott KA (1965) Amino acids released from the cerebral cortex in relation to its state of activation. Science 147:1448-1449.

Ji D, Wilson MA (2007) Coordinated memory replay in the visual cortex and hippocampus during sleep. Nat Neurosci 10:100-107.

John J, Ramanathan L, Siegel JM (2008) Rapid changes in glutamate levels in the posterior hypothalamus across sleep-wake states in freely behaving rats. Am J Physiol Regul Integr Comp Physiol 295:R2041-R2049.

Jones BE (2005) From waking to sleeping: neuronal and chemical substrates. Trends Pharmacol Sci 26:578-586.

Karadzic V, Micic D, Rakic L (1971) Alterations of free amino acids concentrations in cat brain induced by rapid eye movement sleep deprivation. Experientia 27:509-511.

Kékesi KA, Dobolyi A, Salfay O, Nyitrai G, Juhász G (1997) Slow wave sleep is accompanied by release of certain amino acids in the thalamus of cats. Neuroreport 8:1183-1186.

Kennedy C, Gillin JC, Mendelson W, Suda S, Miyaoka M, Ito M, Nakamura RK, Storch FI, Pettigrew K, Mishkin M, Sokoloff L (1982) Local cerebral glucose utilization in non-rapid eye movement sleep. Nature 297:325-327.

Kodama T, Honda Y (1999) Acetylcholine and glutamate release during sleep-wakefulness in the pedunculopontine tegmental nucleus and norepinephrine changes regulated by nitric oxide. Psychiatry Clin Neurosci 53:109-111.

Kodama T, Lai YY, Siegel JM (1998) Enhanced glutamate release during REM sleep in the rostromedial medulla as measured by in vivo microdialysis. Brain Res 780:178-181.

Léna I, Parrot S, Deschaux O, Muffat-Joly S, Sauvinet V, Renaud B, SuaudChagny MF, Gottesmann C (2005) Variations in extracellular levels of dopamine, noradrenaline, glutamate, and aspartate across the sleep-wake cycle in the medial prefrontal cortex and nucleus accumbens of freely moving rats. J Neurosci Res 81:891-899.

Lopez-Rodriguez F, Medina-Ceja L, Wilson CL, Jhung D, Morales-Villagran A (2007) Changes in extracellular glutamate levels in rat orbitofrontal cortex during sleep and wakefulness. Arch Med Res 38:52-55.

Madsen PL, Schmidt JF, Wildschiødtz G, Friberg L, Holm S, Vorstrup S, Lassen NA (1991) Cerebral O2 metabolism and cerebral blood flow in humans during deep and rapid-eye-movement sleep. J Appl Physiol 70:2597-2601.

Mangano RM, Schwarcz R (1983) Chronic infusion of endogenous excitatory amino acids into rat striatum and hippocampus. Brain Res Bull $10: 47-51$.
Maquet P (1995) Sleep function(s) and cerebral metabolism. Behav Brain Res 69:75-83.

Mark J, Godin Y, Mandel P (1969) Biosynthesis of aspartic, glutamic, gamma-aminobutyric acids and glutamine in brain of rats deprived of total sleep or paradoxical sleep. J Neurochem 16:1263-1272.

Nicoll RA, Madison DV (1982) General anesthetics hyperpolarize neurons in the vertebrate central nervous system. Science 217:1055-1057.

Noda H, Adey WR (1973) Neuronal activity in the association cortex of the cat during sleep, wakefulness and anesthesia. Brain Res 54:243-259.

Oldenziel WH, Dijkstra G, Cremers TI, Westerink BH (2006) In vivo monitoring of extracellular glutamate in the brain with a microsensor. Brain Res 1118:34-42.

Pomerleau F, Day BK, Huettl P, Burmeister JJ, Gerhardt GA (2003) Real time in vivo measures of L-glutamate in the rat central nervous system using ceramic-based multisite microelectrode arrays. Ann N Y Acad Sci 1003:454-457.

Regan RF, Choi DW (1991) Glutamate neurotoxicity in spinal cord cell culture. Neuroscience 43:585-591.

Richards CD, Strupinski K (1986) An analysis of the action of pentobarbitone on the excitatory postsynaptic potentials and membrane properties of neurones in the guinea-pig olfactory cortex. Br J Pharmacol 89:321-325.

Rosenberg PA, Amin S, Leitner M (1992) Glutamate uptake disguises neurotoxic potency of glutamate agonists in cerebral cortex in dissociated cell culture. J Neurosci 12:56-61.

Rothstein JD, Dykes-Hoberg M, Pardo CA, Bristol LA, Jin L, Kuncl RW, Kanai Y, Hediger MA, Wang Y, Schielke JP, Welty DF (1996) Knockout of glutamate transporters reveals a major role for astroglial transport in excitotoxicity and clearance of glutamate. Neuron 16:675-686.

Rutherford EC, Pomerleau F, Huettl P, Strömberg I, Gerhardt GA (2007) Chronic second-by-second measures of L-glutamate in the central nervous system of freely moving rats. J Neurochem 102:712-722.

Sallanon-Moulin M, Touret M, Didier-Bazes M, Roudier V, Fages C, Tardy M, Jouvet M (1994) Glutamine synthetase modulation in the brain of rats subjected to deprivation of paradoxical sleep. Brain Res Mol Brain Res 22:113-120.

Santello M, Volterra A (2008) Synaptic modulation by astrocytes via $\mathrm{Ca}(2+)$ dependent glutamate release. Neuroscience. Advance online publication. Retrieved December 30, 2008. doi:10.1016/j.neuroscience.2008.03.039.

Sem'yanov AV (2005) Diffusional extrasynaptic neurotransmission via glutamate and GABA. Neurosci Behav Physiol 35:253-266.

Steriade M, Timofeev I, Grenier F (2001) Natural waking and sleep states: a view from inside neocortical neurons. J Neurophysiol 85:1969-1985.

Tang X, Yang L, Sanford LD (2007) Sleep and EEG spectra in rats recorded via telemetry during surgical recovery. Sleep 30:1057-1061.

Timmerman W, Westerink BH (1997) Brain microdialysis of GABA and glutamate: what does it signify? Synapse 27:242-261.

Tzingounis AV, Wadiche JI (2007) Glutamate transporters: confining runaway excitation by shaping synaptic transmission. Nat Rev Neurosci 8:935-947.

van der Zeyden M, Oldenziel WH, Rea K, Cremers TI, Westerink BH (2007) Microdialysis of GABA and glutamate: analysis, interpretation and comparison with microsensors. Pharmacol Biochem Behav 90:135-147.

Vyazovskiy VV, Cirelli C, Tononi G, Tobler I (2008a) Cortical metabolic rates as measured by 2-deoxyglucose-uptake are increased after waking and decreased after sleep in mice. Brain Res Bull 75:591-597.

Vyazovskiy VV, Cirelli C, Pfister-Genskow M, Faraguna U, Tononi G (2008b) Molecular and electrophysiological evidence for net synaptic potentiation in wake and depression in sleep. Nat Neurosci 11:200-208.

Wadiche JI, Arriza JL, Amara SG, Kavanaugh MP (1995) Kinetics of a human glutamate transporter. Neuron 14:1019-1027.

Wan X, Mathers DA, Puil E (2003) Pentobarbital modulates intrinsic and GABA-receptor conductances in thalamocortical inhibition. Neuroscience 121:947-958. 